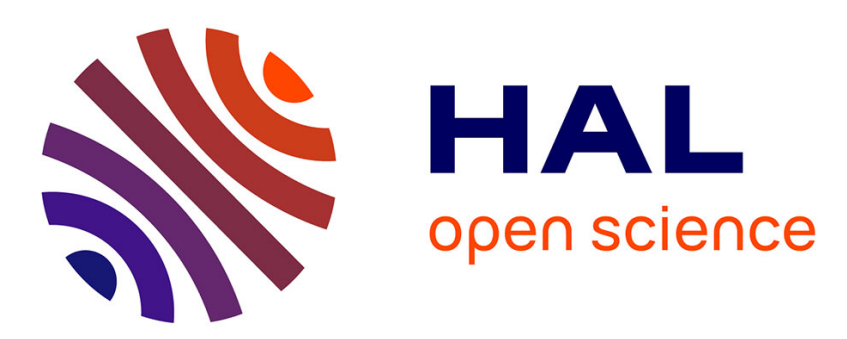

\title{
THE TWO PHOTON TWO INTERMEDIATE STATE DECAY OR THE EFFECT OF COHERENT RELAXATION ON MÖSSBAUER SPECTRA
}

\author{
E. Kankeleit, A. Körding
}

\section{- To cite this version:}

E. Kankeleit, A. Körding. THE TWO PHOTON TWO INTERMEDIATE STATE DECAY OR THE EFFECT OF COHERENT RELAXATION ON MÖSSBAUER SPECTRA. Journal de Physique Colloques, 1976, 37 (C6), pp.C6-65-C6-70. 10.1051/jphyscol:1976616 . jpa-00216679

\section{HAL Id: jpa-00216679 https://hal.science/jpa-00216679}

Submitted on 1 Jan 1976

HAL is a multi-disciplinary open access archive for the deposit and dissemination of scientific research documents, whether they are published or not. The documents may come from teaching and research institutions in France or abroad, or from public or private research centers.
L'archive ouverte pluridisciplinaire HAL, est destinée au dépôt et à la diffusion de documents scientifiques de niveau recherche, publiés ou non, émanant des établissements d'enseignement et de recherche français ou étrangers, des laboratoires publics ou privés. 


\title{
THE TWO PHOTON TWO INTERMEDIATE STATE DECAY OR THE EFFECT OF COHERENT RELAXATION ON MÖSSBAUER SPECTRA
}

\author{
E. KANKELEIT and A. KÖRDING \\ Institut für Kernphysik, Technische Hochschule Darmstadt, Germany
}

\begin{abstract}
Résumé. - La désintégration cohérente d'un système atomique et nucléaire à couplage faible, tous deux excités simultanément, est analysée et la fréquence qui en résulte, ainsi que le spectre Mössbauer, sont calculés. La relation avec un traitement semi-classique sans spin est démontrée.
\end{abstract}

\begin{abstract}
The coherent decay of a weakly coupled atomic and nuclear system both simultaneously excited is analyzed and the resulting frequency and Mössbauer spectrum calculated. The relation to a semiclassical treatment for a spinless system is demonstrated.
\end{abstract}

1. Introduction : coherent relaxation. - One of the interesting applications of the Mössbauer (MB) effect concerns the emission spectroscopy, the study of unusual chemical configurations produced by the change of atomic charge and ionization after beta decay. If these configurations are unstable and decay in times comparable to the nuclear lifetime one expects in the emission spectrum a superposition of spectra belonging to the stable and unstable electronic configurations. The relative contribution will depend of course on the ratio on the electronic lifetime to the nuclear lifetime. In a time differential experiment one expects that the unstable configurations contribute most strongly in the early time windows while the stable configurations will finally prevail in the late time windows. The spectra therefore will show modifications which go beyond that expected from the time filtering.

Many experimentalists have looked for these effects since the beginning of $\mathrm{MB}$ spectroscopy. But only recently good evidence for dynamic aftereffects was found in rather complex molecular configurations [1-4]. The necessary condition hard to meet for observing such a coherent relaxation is that the excited atomic and nuclear systems have to have comparable lifetimes. In most cases the MB spectroscopist is not confronted with such a situation because the atomic transitions occur in the subnanosecond domain and the source is for the main part of the nuclear lifetime in its groundstate. Closely related to the coherent relaxation is the well known stochastic relaxation of a hf split source. Only in this case the phases of atomic transitions are uncorrelated to the nuclear emission process. Also the dynamic relaxation of a lattice excited by the beta recoil, by which the emission line is modified, is connected to the coherent relaxation process [5].
We will consider here an idealized situation in which only one atomic configuration may be excited by a prompt preceding decay which also populates the excited MB state. Following the evolution in time for the two decay processes we will ask for the MB spectrum expected if the atomic system (shortly atom) is weakly coupled to the nucleus by a hyperfine interaction. This is a problem which actually should be of importance also in other fields of physics. In the decay of a muonic atom, for instance, there is a weak monopole coupling of the muon to the electronic shell which is excited by Auger transitions [6, 7]. In angular correlation experiments the excited atom may produce a coherent repopulation of hyperfine states which should be different to the usual stochastic one. Also under certain conditions one expects this effect to be existing in nuclear reactions. But so far, this coherent relaxation effect has only been seen in the MB field.

The problem of decay of two weakly coupled systems is identical to that of a two photon two in ermediate state decay. It was touched upon in the very early papers by Weisskopf and Wigner [8, 9]. Later Goldhaber and Watson [10] derived a very general formula which includes this case. There are some semiclassical attempts to derive an expression for the emission spectrum [3, 11, 12]. Unfortunately they are all at variance with each other. But one of these [12] is in agreement with the more basic derivation which we present here. Because of its simplicity it may be justified to repeat briefly the basic idea of this semiclassical picture.

We assume in a Gedankenexperiment to have three detectors. The one detects the preceding radiation (beta particle, for instance) and establishes the time zero for the population of the excited states of nucleus and 
atom. In a delayed coincidence set up a second detector measures (neglecting the time of flight) the time $T^{\prime}$ of the atomic photon. Only if this happens at time $T^{\prime} \pm \Delta T^{\prime}$ we will open the MB detector for times long compared to the nuclear lifetime. This way we know that the amplitude leaving the nucleus has till time $T^{\prime}$ a frequency corresponding to that of the excited atom and a decay constant $\Gamma=\Gamma_{n}+\Gamma_{a}$, the sum of nuclear and atomic decay constants. After time $T^{\prime}$, the decay corresponds to the groundstate atomic configuration and decay constant $\Gamma_{n}$. The intensity in the MB detector for these events is given by the absolute squared amplitude behind the absorber. If we sum up all the measurements for different $T^{\prime}$ we get the final $\mathrm{MB}$ spectrum. The summation corresponds to an integration over $T^{\prime}$ including a factor $\Gamma_{a} \mathrm{e}^{-\Gamma a T^{\prime}}$ which is just the probability for the intensity in the photon counter. The result finally shows in the spectrum two lines, the one broadened, the other of natural width and an interference pattern which will be discussed later.

Even though one may argue that such an approach is as fundamental as a fully quantum mechanical one, it certainly lacks of taking correctly the angular momentum states and photon polarizations into account. The question of phase change and helicity change at the moment of atomic decay remains unclear in the semiclassical picture of spinless photons. It is the purpose of this report to derive a quantum mechanical formula for the MB spectrum first for spinless photons and to show that in that case the semiclassical approach is correct. Finally we include the angular momentum states and discuss under which conditions the interference occurs.

2. Amplitude for a two photon two intermediate state decay. - The hamiltonian for our problem can be written as

$$
\mathscr{H}=\mathscr{H}_{n}+\mathscr{H}_{a}+\mathscr{H}_{n a}+\mathscr{H}_{\mathrm{p}}+\mathscr{H}_{\mathrm{p} a}+\mathscr{H}_{\mathrm{p} n}
$$

which includes the hamiltonians for the isolated systems of nucleus $\mathscr{H}_{n^{\prime}}$ atom $\mathcal{H}_{a^{\prime}}$ photons $\mathcal{H}_{\mathrm{p}}$ and their interactions $\mathscr{H}_{n a^{\prime}}, \mathscr{H}_{\mathrm{p} n}$ and $\mathscr{H}_{\mathrm{pa}}$. We neglect the interaction with the lattice and the recoil. In figure 1 the

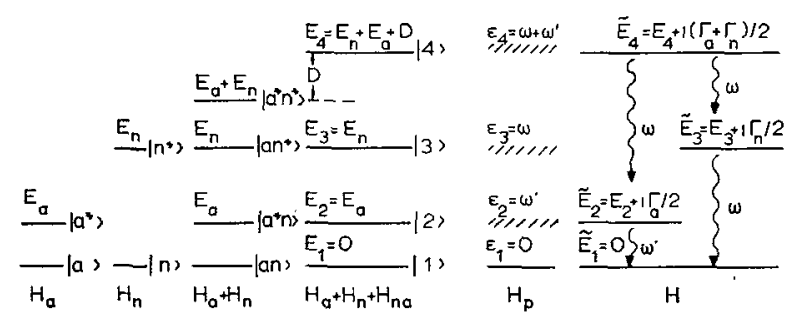

Fig. 1. - Energy diagrams for the atomic system, the nucleus and photons and their interaction. $E_{a}$ and $E_{n}$ are the excitation energies in state $\left|a^{*}\right\rangle$ and $\left|n^{*}\right\rangle$ of atom and nucleus, respectively. The interaction hamiltonian $\mathfrak{H}_{n a}$ produces an isomer shift $D$ in state $|4\rangle$ only. From the continuous photon spectrum those $\varepsilon$ values are indicated which participate in the decay of the four level-system. energy diagrams for the free and coupled systems are indicated. For the moment we assume $\mathcal{H}_{n a}$ to be of monopole type and contributing only in state 4, giving rise to a shift $\mathrm{D}$ in this state. To simplify the formulas a number of notations are introduced as shown in figure 1. The essential point is that the excited state $|4\rangle=\left|n^{*} a^{*}\right\rangle$ can decay via two channels. The nuclear photon $\omega$ may be emitted first, populating state $|2\rangle=\left|n a^{*}\right\rangle$ from which the atomic photon $\omega^{\prime}$ is then emitted. The other channel proceeds by first emitting $\omega^{\prime}$, then the nuclear excited state $|3\rangle=\left|n^{*} a\right\rangle$ decays with photon $\omega$. The decay width in state 4 is $\Gamma_{4}=\Gamma_{a}+\Gamma_{\mathrm{n}}$ the sum of atomic and nuclear decay probabilities.

For the interaction hamiltonians with the photonfield we write

$$
\begin{aligned}
& \mathscr{H}_{\mathrm{p} n}=\int \mathrm{d} \omega M_{n}^{\lambda}(\omega) a_{\lambda}^{+}+\text {h. c. } \\
& \mathfrak{H}_{\mathrm{p} a}=\int \mathrm{d} \omega^{\prime} M_{a}^{\lambda^{\prime}}\left(\omega^{\prime}\right) a_{\lambda^{\prime}}^{+}+\text {h. c. }
\end{aligned}
$$

where $\lambda$ characterizes the quantum numbers of the multipole field. The multipole operators $M_{n}^{\lambda}(\omega)$, $\mathbf{M}_{a}^{\lambda^{\prime}}\left(\omega^{\prime}\right)$ are effective only for the nuclear or atomic states, respectively, $a_{\lambda}^{+}$are the photon creation operator. Following in line of Weisskopf and Wigner [8, 9] and Goldhaber, Watson [10] we write with all the assumptions and approximations discussed in these references the decay amplitude for the two channels.

$$
\begin{aligned}
a\left(\omega, \omega^{\prime}\right) & =\frac{\left\langle 1\left|M_{a}\left(\omega^{\prime}\right)\right| 2><2\left|M_{n}(\omega)\right| 4\right\rangle}{\left(\varepsilon_{4}-\widetilde{E}_{4}\right)\left(\varepsilon_{2}-\widetilde{E}_{2}\right)}+ \\
+ & \frac{\left\langle 1\left|M_{n}(\omega)\right| 3><3\left|M_{a}\left(\omega^{\prime}\right)\right| 4>\right.}{\left(\varepsilon_{4}-\widetilde{E}_{4}\right)\left(\varepsilon_{3}-\widetilde{E}_{3}\right)}
\end{aligned}
$$

Now

$\varepsilon_{4}-\widetilde{E}_{4}=\omega+\omega^{\prime}-\left(E_{n}+E_{a}+D+i \frac{\Gamma_{n}+\Gamma_{a}}{2}\right)$

$\varepsilon_{2}-\widetilde{E}_{2}=\omega^{\prime}-\left(E_{a}+i \frac{\Gamma_{a}}{2}\right)$

$\varepsilon_{3}-\widetilde{E}_{3}=\omega-\left(E_{n}+i \frac{\Gamma_{n}}{2}\right)$

The two terms are significant in size only if the sum of the two frequencies is about $E_{4}=E_{a}+E_{\mathrm{n}}+D$ as well as $\omega^{\prime} \simeq E_{a}$ for the first term and $\omega \simeq E_{n}$ for the second term. Because the level width and the shift $D$ are very small compared to $E_{n}$ and $E_{a}$ we may ignore the energy dependence in the transition matrix elements. For the moment we will furthermore ignore the spin of all particles involved. Then we can write with

$$
\left\langle 1\left|M_{a}\right| 2\right\rangle=\left\langle 3\left|M_{a}\right| 4\right\rangle=M_{a}
$$


and

$$
\begin{aligned}
&\left.<1\left|M_{n}\right| 3\right\rangle=\left\langle 2\left|M_{n}\right| 4\right\rangle=M_{n} \\
& a\left(\omega, \omega^{\prime}\right)= \frac{M_{a} M_{n}}{\left(\varepsilon_{4}-\widetilde{E}_{4}\right)}\left(\frac{1}{\varepsilon_{2}-\widetilde{E}_{2}}+\frac{1}{\varepsilon_{3}-\widetilde{E}_{3}}\right) \\
&=\frac{M_{a} M_{n}}{\omega+\omega^{\prime}-\widetilde{E}_{4}}\left(\frac{1}{\omega^{\prime}-\widetilde{E}_{2}}+\frac{1}{\omega-\widetilde{E}_{3}}\right)
\end{aligned}
$$

We will first ask for the probability to find a photon of energy $\omega$ from the nuclear decay if the atomic photon $\omega^{\prime}$ is not detected. This is

$$
I(\omega)=\int \mathrm{d} \omega^{\prime}\left|a\left(\omega, \omega^{\prime}\right)\right|^{2} .
$$

After a simple but lengthy calculation using the residue theorem we obtain with

$$
\left|M_{a}\right|^{2} 2 \pi=\Gamma_{a} \quad \text { and } \quad\left|M_{n}\right|^{2} 2 \pi=\Gamma_{n}
$$

$$
\begin{aligned}
I(\omega)=\frac{1}{2 \pi} \frac{\Gamma_{n} \Gamma_{a}}{\Gamma_{n}+\Gamma_{a}}\left\{\frac{1}{\left(\omega-E_{a}\right)^{2}+\left(\Gamma_{n} / 2\right)^{2}}+\frac{\Gamma_{n}+2 \Gamma_{a}}{\Gamma_{a}} \frac{1}{\left(\omega-\left(E_{n}+D\right)\right)^{2}+\left(\frac{\Gamma_{n}+2 \Gamma_{a}}{2}\right)^{2}}\right. \\
\left.-2 \frac{\left(\omega-E_{n}\right)\left(\omega-\left(E_{n}+D\right)\right)-\frac{\Gamma_{n}}{2} \frac{2 \Gamma_{a}+\Gamma_{n}}{2}}{\left[\left(\omega-E_{n}\right)^{2}+\left(\frac{\Gamma_{n}}{2}\right)^{2}\right]\left[\left(\omega-\left(E_{n}+D\right)\right)^{2}+\left(\frac{2 \Gamma_{a}+\Gamma_{n}}{2}\right)^{2}\right]}\right\}
\end{aligned}
$$

This is the same result as derived in the semiclassical approach. The first term corresponds to an unshifted Lorentzian of natural width. Vaguely speaking it is the part belonging to times after the atom has decayed. It is the only term surviving if $\Gamma_{a} \gg \Gamma_{n} \mathrm{i}$. e. for short lifetimes of the atom. The opposite is true for the second term. For this the Lorentzian is shifted by $D$, and is additionally broadened by $2 \Gamma_{a}$. The third term clearly results from the interference between the two decay channels. It disappears, if there is no overlap between the two lines. We will now ask for the amplitude of the atomic photon $\omega^{\prime}$ to be at a large distance $r^{\prime}$ at time $t>0$. Assuming an s-wave emission it turns out that the ingoing part of the Besselfunction disappears and we get

$$
\begin{aligned}
a\left(\omega, r^{\prime}, t\right)= & \int \mathrm{d} \omega \mathrm{e}^{-i t \omega^{\prime}} \frac{e^{i r^{\prime} \omega^{\prime}}}{2 \pi i \sqrt{2} r^{\prime}\left(\omega+\omega^{\prime}-\widetilde{E}_{4}\right)}\left(\frac{M_{n} M_{a}}{\omega-\widetilde{E}_{3}}+\frac{1}{\omega^{\prime}-\widetilde{E}_{2}}\right) \\
= & \frac{M_{n} M_{a}}{\sqrt{2} r^{\prime}} \mathrm{e}^{-i\left(t-r^{\prime}\right) \widetilde{E}_{2}} \theta\left(t-r^{\prime}\right)\left\{\frac{1}{\omega-\left(\widetilde{E}_{4}-\widetilde{E}_{2}\right)}+\mathrm{e}^{i\left(t-r^{\prime}\right)\left(\omega-\left(\widetilde{E}_{4}-\widetilde{E}_{2}\right)\right)} .\right. \\
& \left.\cdot\left(\frac{1}{\omega-\widetilde{E}_{3}}-\frac{1}{\omega-\left(\widetilde{E}_{4}-\widetilde{E}_{2}\right)}\right)\right\} .
\end{aligned}
$$

We recognize that the wave packet can reach the position $r^{\prime}$ not faster than speed of light. It arrives at time $t=r^{\prime}$ when the step function $\theta\left(t-r^{\prime}\right)$ becomes unity. Next we will ask when the wave packet of the nuclear photon $\omega$ arrives at point $r$.

$$
\begin{aligned}
a\left(r, r^{\prime}, t\right) & =\int \mathrm{d} \omega \mathrm{e}^{i t \omega} \frac{\mathrm{e}^{i r \omega}}{2 \pi i \sqrt{2} r} a\left(\omega, r^{\prime}, t\right) \\
& =\frac{1}{2} \frac{M_{n} M_{a}}{r r^{\prime}} \mathrm{e}^{-i\left(t-r^{\prime}\right) \tilde{E}_{2}} \mathrm{e}^{-i(t-r) \tilde{E}_{3}} \cdot \mathrm{e}^{-i(t-r) D}, \\
\mathrm{e}^{-i\left(t-r^{\prime}\right) D}, & t>r>r^{\prime} \\
0, & t>r^{\prime}>r \\
\exp \left[-i T^{\prime}\left(E_{a}+i \frac{\Gamma_{a}}{2}\right)\right] \exp \left[-i T\left(E_{n}+D+i \frac{\Gamma_{n}}{2}\right)\right], & T<r^{\prime} t<
\end{aligned}
$$

This wave function does indeed reflect the semiclassical picture to some extent. If we consider $T^{\prime}=t-r^{\prime}$ and $T=t-r$ as variables we realize that first, for $T<T^{\prime}$, the nuclear photon is emitted with an energy $E_{n}+D$. This energy is changed to $E_{n}$ for $T>T^{\prime}$ when the atomic photon has already arrived. In particular we see now 
that at the point $T^{\prime}=T$ the amplitude remains steadily, no change in phase occurs. A more detailed discussion of these points will be presented by one of the authors (A. K.) in a forthcoming paper.

Finally we want to determine the intensity in the MB detector behind a resonance absorber. This absorber moves with a velocity according to an energy shift $V$ and a thickness $t_{a}$ as usually defined. We arrive the easiest way at the velocity spectrum by starting from eq. (4).

The amplitude behind the absorber is given by [13]

$$
\widehat{a}\left(\omega, \omega^{\prime}, V\right)=\exp \left[\frac{i t_{a} \Gamma_{n} / 4}{\omega-\left(\widetilde{E}_{3}+V\right)}\right] a\left(\omega, \omega^{\prime}\right),
$$

Because as neither for the atomic nor the nuclear photon the energy is determined we get

$$
I(V)=\int \mathrm{d} \omega^{\prime} \int \mathrm{d} \omega\left|\hat{a}\left(\omega, \omega^{\prime}, V\right)\right|^{2} .
$$

We may of course use eq. (7) as well and derive the amplitude

$$
\widehat{a}\left(\omega, r^{\prime}, t\right)=\exp \left[\frac{i t_{a} \Gamma_{n} / 4}{\omega-\left(\widetilde{E}_{2}+V\right)}\right] a\left(\omega, r^{\prime}, t\right) .
$$

The intensity is now with $T^{\prime}=t-r^{\prime}$ given by

$$
I(V)=\frac{\left|M_{n}\right|^{2}}{2 r^{\prime 2}} \int_{0}^{\infty} \mathrm{d} T^{\prime}\left|M_{a}\right|^{2} \mathrm{e}^{-\Gamma a T^{\prime}} \int \mathrm{d} \omega \exp \left[\frac{-t_{a}\left(\Gamma_{n} / 2\right)^{2}}{\left(\omega-\left(E_{n}+V\right)\right)^{2}+\left(\frac{\Gamma_{n}}{2}\right)^{2}}\right]|\{\}|^{2}
$$

The curly brackets are those of eq. (7). Here we see by which way the weighting factor $\Gamma_{a} \mathrm{e}^{-i T^{\prime} T a}$ appears and justifies again the semiclassical procedure.

For a time differential experiment one might start from eq. (8) in which the amplitude $\hat{a}\left(\omega, r^{\prime}, t\right)$ is inserted in the integral instead of $a\left(\omega, r^{\prime}, t\right)$. We thus arrive at $\widehat{a}\left(T, T^{\prime} v\right)$. The intensity at the MB detector as a function of delay time $T$ is given by

$$
I(T \cdot V)=\int_{0}^{\infty} \mathrm{d} T^{\prime}\left|\widehat{a}\left(T, T^{\prime}, V\right)\right|^{2}
$$

which has been calculated in ref. [12].

For the sake of demonstrating the typical features of the shape of transmission spectra we quote the result for a thin absorber

$$
\begin{aligned}
M(V)=\frac{1}{\pi}\left[\frac{\Gamma_{n}}{\Gamma_{a}+\Gamma_{n}} \frac{\Gamma_{a}+\Gamma_{n}}{(V-D)^{2}+\left(\Gamma_{a}+\Gamma_{n}\right)^{2}}+\frac{\Gamma_{a}}{\Gamma_{a}+\Gamma_{n}} \frac{\Gamma_{n}}{V^{2}+\Gamma_{n}^{2}}\right. & \\
& \left.-\frac{\Gamma_{a} \Gamma_{n}}{\Gamma_{a}+\Gamma_{n}} \frac{(V-D) V-\Gamma_{n}\left(\Gamma_{a}+\Gamma_{n}\right)}{\left[V^{2}+\Gamma_{n}^{2}\right]\left[(V-D)^{2}+\left(\Gamma_{a}+\Gamma_{n}\right)^{2}\right]}\right]
\end{aligned}
$$

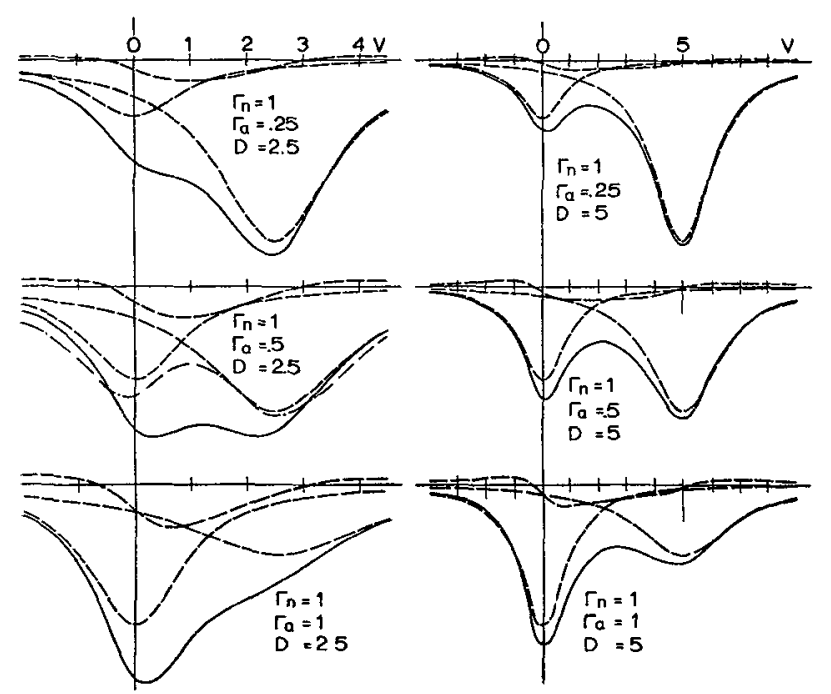

FIG. 2. - Mössbauer spectra $M(V)$, demonstrating the effect of coherent relaxation in a spinless system. They result from a superposition of a natural width $\left(2 \Gamma_{n}\right)$ Lorentzian at zero velocity, a broadened one at $D$ and an interference part. In the figure with $\Gamma_{a}=0.5$ and $D=2.5$ the dot-dashed curve indicates the drastic effect if the phase is changed by $180^{\circ}$ at the moment of atomic decay. 
and present some spectra as shown in figure 2. We recognize the broadening of the 4-2 line shifted by $D$ and the sizable contribution of the interference effect. In one of the figures the dash dotted line indicates the drastic effect if the phase after the atomic decay is changed by $180^{\circ}$. How this may come about will be discussed now.

3. Consideration of angular momentum. - In the model described so far we have neglected the photon polarizations. For the question of interference it is of course necessary to consider these. Only if the polarization of the nuclear photon is the same before and after the atomic decay an interference effect is to be expected. It will be a very difficult task to derive general formulas as it was done for the stochastic relaxation process. A detailed knowledge is needed hete in particular about the atomic system and its decay. From a MB experiment we can derive the hf splittings in the states 1 to 4 from the superposition of two spectra belonging to the atomic ground- and excited state, respectively. Between certain hf components a smearing of the spectra may indicate an interference effect. It is the object of this last part to check under which condition this interference might arrive.

For doing this we will consider the coupled nuclear and atomic system in isotropic space. Thus the angular momenta $j_{n}$ and $j_{a}$ are coupled to total spin $\mathbf{J}=\mathbf{j}_{a}+\mathbf{j}_{n}$. Such a situation is prevailing for a muonic atom with its interaction of inner electron shells. In the general case we have to expect a hf interaction in all four states, thus that to each $J$ the degeneracy is lifted. For instance there will be different $J_{2 v}$ from $\left|j_{n}-j_{a^{*}}\right|$ to $\left|j_{n}+j_{n^{*}}\right|$ with energy $E_{2 v}$ and similarly there will by different $J_{3 \mu}$. We will resign to characterize the initial state 4 and find state 1 by additional indices.

The partial widths for the nuclear and atomic transitions of multipolarity $l, l^{\prime}$ and parity $\pi, \pi^{\prime}$ are given by

$$
\begin{aligned}
& \Gamma_{n}^{l}=\frac{2 \pi}{2 j_{n^{*}}+1}\left|<j_{n}\left\|M^{l \pi}(\omega)\right\| j_{n^{*}}>\right|^{2} \leqslant \Gamma_{n} \\
& \Gamma_{a}^{l^{\prime}}=\frac{2 \pi}{2 j_{a^{*}}+1}\left|<j_{a}\left\|M^{l^{\prime} \pi^{\prime}}\left(\omega^{\prime}\right)\right\| j_{a^{*}}>\right|^{2} \leqslant \Gamma_{a} .
\end{aligned}
$$

We need furthermore the reduced matrix elements for the coupled system, for instance

$$
\begin{aligned}
<J_{1} j_{n}\left\|M^{l \pi}(\omega)\right\| J_{3 \mu} j_{n^{*}} & >\equiv<J_{1}\left\|M^{l \pi}\right\| J_{3 \mu}>= \\
& =(-1)^{J_{3 \mu}+l+j_{n}+j_{a}} \sqrt{\left(2 J_{1}+1\right)\left(2 J_{3_{\mu}}+1\right)}\left\{\begin{array}{c}
j_{a} j_{n} J_{1} \\
l J_{3_{\mu}} j_{n^{*}}
\end{array}\right\}<j_{n}\left\|M^{l \pi}(\omega)\right\| j_{a}>.
\end{aligned}
$$

Now we may start from eq. (3) and write the energy distribution in complete form for a transition from one of the states 4 to one of the states 1 summed over the polarizations and directions of the two photons

$$
\begin{aligned}
& I\left(\omega l \pi, \omega^{\prime} l^{\prime} \pi^{\prime}, J_{4} \rightarrow J_{1}\right)=\frac{1}{2 J_{4}+1} \frac{1}{\left|\omega+\omega^{\prime}-\left(\widetilde{E}_{4}-E_{1}\right)\right|^{2}} \\
& \quad \times\left[\sum_{\mu}\left|\frac{<J_{1}\left\|M^{l \pi}\right\| J_{3 \mu}><J_{3 \mu}\left\|M^{l^{\prime} \pi^{\prime}}\right\| J_{4}>}{\sqrt{2 J_{3 \mu}+1}\left(\omega-\widetilde{E}_{3 \mu}+E_{1}\right)}\right|^{2}\right. \\
& \quad+\sum_{v}\left|\frac{<J_{1}\left\|M^{l^{\prime} \pi^{\prime}}\right\| J_{2 v}><J_{2 v}\left\|M^{l \pi}\right\| J_{4} \geq\left.\right|^{2}}{\sqrt{2 J_{2 v}+1}\left(\omega^{\prime}-\widetilde{E}_{2 v}+E_{1}\right)}\right|^{2} \\
& +\sum_{v \mu} U\left(J_{1} J_{4} l l^{\prime} ; J_{2 v} J_{3 \mu}\right) . \\
& \quad 2 \operatorname{Re}\left\{\frac{\left.\left.<J_{1}\left\|M^{l \pi}\right\| J_{3 \mu}><J_{3 \mu}\left\|M^{i^{\prime} \pi^{\prime}}\right\| J_{4}><J_{1}\left\|M^{i^{\prime} \pi^{\prime}}\right\| J_{2 v}><J_{2 v}\left\|M^{l \pi}\right\| J_{4} \geq\right\}\right]}{\sqrt{2 J_{3 \mu}+1} \sqrt{2 J_{2 v}+1}\left(\omega-\widetilde{E}_{3 \mu}+E_{1}\right)\left(\omega^{\prime}-\widetilde{E}_{2 v}+E_{1}\right)^{*}}\right.
\end{aligned}
$$

Finally we are interested only in the energy spectrum of $\omega$, which by help of eq. (12) and (13) is given by

$$
\begin{aligned}
I\left(\omega l \pi, l^{\prime} \pi^{\prime}, J_{4} \rightarrow J_{1}\right)=\left(2 j_{a^{*}}\right. & +1)\left(2 j_{n^{*}}+1\right)\left(2 J_{1}+1\right) \frac{\Gamma_{a}^{l^{\prime}} \Gamma_{n}^{l} \frac{1}{\Gamma_{a} \Gamma_{n}} \frac{1}{2 \pi} \cdot}{\Gamma_{n}}\left(2 J_{3 \mu}+1\right)\left\{\begin{array}{l}
J_{1} l J_{3 \mu} \\
j_{n^{*}} j_{a} j_{n}
\end{array}\right\}^{2}\left\{\begin{array}{l}
J_{3 \mu} l^{\prime} J_{4} \\
j_{a^{*}} j_{n^{*}} j_{a}
\end{array}\right\}^{2} \\
& \cdot\left[\frac{\Gamma_{a}}{\Gamma_{a}+\Gamma_{n}} \sum_{\mu} \frac{\Gamma_{n}}{\omega-\left.\left(E_{3 \mu}-E_{1}+i \frac{\Gamma_{n}}{2}\right)\right|^{2}}\right. \\
& +\frac{\Gamma_{n}}{\Gamma_{n}+\Gamma_{a}} \sum_{v} \frac{\Gamma_{n}+2 \Gamma_{a}}{\left|\omega-\left(E_{4}-E_{2 v}+i \frac{\Gamma_{n}}{2}\right)\right|^{2}}\left(2 J_{2 v}+1\right)\left\{\begin{array}{l}
J_{1} l^{\prime} J_{2 v} \\
j_{a^{*}} j_{n} j_{a}
\end{array}\right\}^{2}\left\{\begin{array}{l}
J_{2 v} l J_{4} \\
j_{n^{*}} j_{a^{*}} j_{n}
\end{array}\right\}^{2}
\end{aligned}
$$




$$
\begin{aligned}
& -2 \frac{\Gamma_{a} \Gamma_{n}}{\Gamma_{a}+\Gamma_{n}}(-1)^{J_{1}+J_{4}+j a+j n+j a^{*}+j n^{*}} . \\
& \cdot \sum_{v \mu} 2 \operatorname{Re}\left\{\frac{U\left(J_{1} J_{4} l l^{\prime} ; J_{2 v} J_{3 \mu}\right) \sqrt{\left(2 J_{2 v}+1\right)\left(2 J_{3 \mu}+1\right)}}{\left[\omega-\left(E_{3 \mu}-E_{1}+i \frac{\Gamma_{n}}{2}\right)\right]\left[\omega-\left(E_{4}-E_{2 v}-i \frac{\Gamma_{n}}{2}\right)\right]} .\right. \\
& \left.\left.\cdot\left\{\begin{array}{c}
J_{1} l J_{3 \mu} \\
j_{n^{*}} j_{a} j_{n}
\end{array}\right\}\left\{\begin{array}{c}
J_{3 \mu} l^{\prime} J_{4} \\
j_{a^{*}} j_{n^{*}} j_{a}
\end{array}\right\}\left\{\begin{array}{c}
J_{1} l^{\prime} J_{2 v} \\
j_{a^{*}} j_{n} j_{a}
\end{array}\right\}\left\{\begin{array}{l}
J_{2 v} l J_{4} \\
j_{n^{*}} j_{a^{*}} j_{n}
\end{array}\right\}\right)\right] .
\end{aligned}
$$

We see that we have for each set of intermediate states again a broadened and an unbroadened Lorentzian and besides the phase factor $U$ the product of both as the interference term.

The phase factor $U$ can be calculated as follows

$$
U\left(J_{1} J_{4} l l^{\prime} ; J_{2 v} J_{3 \mu}\right)=(-1)^{l+l^{\prime}-J_{2 \nu}-J_{3 \mu}} \cdot \sqrt{\left(2 J_{2 v}+1\right)\left(2 J_{3 \mu}+1\right)}\left\{\begin{array}{lll}
l^{\prime} & J_{1} J_{2 v} \\
l & J_{4} J_{3 \mu}
\end{array}\right\} .
$$

It has the property that

$$
\begin{array}{rl}
|U| \leqslant 1 & 1 \\
|U|= & 1 \text { for } l+l^{\prime}=\left|J_{1}-J_{4}\right| \\
& \text { or either } \\
J_{1}, J_{4}=0 & J_{1}+J_{4} \\
& \\
& \text { or either }
\end{array}
$$

4. Conclusion. - We have seen that the MB spectroscopist is confronted with quite a difficult problem. in analyzing the MB spectrum of a coherent relaxation process. In general he will have no knowledge by which multipole transition the atomic system is decaying. From a practical point of view it seems advisable in such a case as it was done by Gal et al. [3] to introduce in a fitting procedure a parameter which takes care of the broadening of those lines belonging to the excited atomic state and adjusting the factor $|U| \leqslant 1$ which multiplies the interference term in eq. (15). Furthermore it may be necessary to consider that after the preceding decay several atomic states including the groundstate may be populated.

\section{References}

[1] Ensling, J., Fitzsimmons, B. W., Gürtich, P. and HasselBACH, K. M., Angew. Chem. 82 (1970) 639.

[2] Alekseev, V. P., Goldanski, V. I., Prusakov, V. E., Nefedev, A. V. and Stukan, R. S., J. E. T. P. Lett. 16 (1972) 43.

[3] Gal, J., Hadari, Z., Bauminger, E. R., Nowik, I. and OfER, S., Solid State Commun. 15 (1974) 1805.

[4] Grimm, R., Gǘllich, P., Link, R., Kankeleit, E., ReicheNBäCHER, W. and WALChER, D., «Int. Conf, on Mössbauer Spectroscopy 》 Cracow (1975);

Grimm, R., Gütlich, P., Kankeleit, E. and Link, R., $J$. Physique Colloq. 37 (1976) C 6-last session.

[5] Harris, S. M., Phys. Rev. 163 (1967) 280.
[6] KANKELEIT, E. and KöRDING, A., Hyperfine Structure and Nuclear Radiations (North Holland Publ.) 1968.

[7] Vogel, P., Phys. Rev. A 8 (1973) 2292.

[8] WeIssKopf, V. and Wigner, E., Z. Phys, 63 (1930) 54.

[9] WeISSKOPF, V. and WIGNER, E., Z. Phys. 65 (1930) 18.

[10] Goldhaber, A. S. and Watson, K. M., Phys. Rev. 160 (1967) 1151.

[11] Triftshäuser, W. and Craig, P. P., Phys. Rev. 162 (1967) 274.

[12] Kankeleit, E., Z. Phys. A 275 (1975) 119.

[13] Lynch, F. J., Holland, R. E., Hamermesh, M., Phvs. Rev $120(1960) 513$. 